\title{
Preferential Solvation of Dextran in Water-Ethanol Mixtures
}

\author{
Hiroyasu NOMURA, ${ }^{*}$ Makoto ONODA, \\ and Yutaka MIYAHARA \\ Department of Chemical Engineering, Faculty of Engineering, \\ Nagoya University, \\ Furo-cho, Chikusa-ku, Nagoya 464, Japan.
}

(Received June 20, 1981)

\begin{abstract}
The amount of water bound on dextran in water-ethanol mixtures was determined by measuring the adiabatic compressibility of dextran samples of molecular weight above $10^{4}$. Similar measurements were also carried out on glucose, maltose, and raffinose, and the results were compared with those of dextran. The partial specific compressibility $\left(\bar{\kappa}_{2}^{0}\right)$ of these saccharides increases with increasing ethanol concentration in solvent and takes a constant value above about $20 \mathrm{wt} \%$ of ethanol. The interpretation for this is that the water molecules bound to solute are removed by ethanol; thus, dextran is hydrated preferentially in water-ethanol mixtures. The viscosity of dextran solutions was measured as a function of ethanol concentration and the molecular weight of the dextran samples. From the values of $\bar{\kappa}_{2}^{0}$ at concentrations of ethanol above about $20 \mathrm{wt} \%$, the compressibility of dextran in solution was estimated to be about $4.0 \times 10^{-11}$ $\mathrm{Pa}^{-1}$. This value was compared with the elastic moduli of glucose, maltose, and dextran, estimated from their bond-stretching and -bending force constant.

KEY WORDS Preferential Solvation / Dextran / Partial Specific Compressibility / Viscosity /Amount of Bound Water / Intrinsic Viscosity /
\end{abstract}

About thirty years ago, Miyahara and Shiio ${ }^{1,2}$ and Shiio $^{3}$ proposed a method for evaluating the amount of water bound on nonelectrolytes and polymers in an aqueous solution on the basis of ultrasonic velocity measurements. Their method has been applied widely for determing the hydration number of biopolymers, such as polysaccharides, ${ }^{4-6}$ and proteins. ${ }^{7,8}$

In most determinations of this hydration number for nonelectrolytes and polymers, the adiabatic compressibility of solute, $\kappa_{2}$, has been assumed to be zero. This assumption has been found to be reasonable for low molecular weight samples, but its application to high polymers is disputable, since polymers are flexible and their compressibility falls in the range from 20 to $30 \times 10^{-11} \mathrm{~Pa}^{-1}$.

For a suspension system, it may be assumed that the compression of the system is the arithmetic sum of the compression of components, because the

\footnotetext{
* To whom correspondence should be addressed.
}

diameter of a suspensoid is smaller than the wavelength of the ultrasonic wave used, but at the same time the diameter of the suspensoid is large enough for the suspensoid to be treated as an independent phase. On the basis of this assumption, Urick ${ }^{9}$ and Wada $^{10}$ estimated the compressibility of a suspensoid from compressibility data for a dispersed phase and dispersion medium.

In order to estimate the compressibility of a solute, $\kappa_{2}$, Miyahara and Shiio ${ }^{1,11,12}$ and Itoh $^{13}$ have extended this assumption to hydrophilic colloids. Moreover, they assumed that the addition of a precipitation agent to solution would result in a decrease in the amount of bound water and, at the precipitation point, hydration would become nil.

In our previous papers, ${ }^{14,15}$ we estimated the amount of bound water (hydration number) of dextran in an aqueous solution by ultrasonic velocity measurements. The results of the molecular weight dependence of the amount of bound water have already been discussed in connection with the 
structural change in water as a result of dissolution of the dextran molecule, as shown by the partial molar heat capacity data of aqueous solution of dextran. ${ }^{15}$ In these papers, the compressibility of solute dextran has been assumed to be zero. As mentioned above, this assumption does not seem entirely sound for high polymers. In addition, the amount of water bound to dextran chains as determined on the basis of this assumption appears too small in comparison with the results on glucose which is the basic unit of a dextran chain.

We applied the alcohol-precipitation method to aqueous dextran solutions and estimated the amount of bound water and the compressibility of dextran chains in solution. For comparison, measurements on aqueous solutions of glucose, maltose, and raffinose were also carried out. The intrinsic viscosity of dextrans in water-ethanol mixtures was determined as a function of the ethanol concentration and the molecular weight of the dextran samples.

\section{DEFINITIONS AND NOTATION ${ }^{16}$}

The limiting partial specific compressibility of a solute, $\bar{\kappa}_{2}{ }^{0}$, is defined by,

$$
\bar{\kappa}_{2}{ }^{0}=-\frac{1}{{\overline{v_{2}}}^{0}}\left(\frac{\partial \bar{v}_{2}}{\partial p}\right)_{\mathrm{s}, 0}
$$

where $\bar{v}_{2}$ is the partial specific volume of the solute and the superscript zero refers to this value at infinite dilution. If a mechanical model (the solvation model) is assumed for a polymer molecule in a dilute solution under static compression, $\bar{\kappa}_{2}{ }^{0}$ can be expressed as,

$$
\bar{\kappa}_{2}^{0}=\kappa_{2}+\frac{n_{\mathrm{s}} v_{1}}{v_{2}}\left(\kappa_{\mathrm{s}}-\kappa_{1}\right)
$$

and

$$
\bar{v}_{2}^{0}=v_{2}
$$

where $\kappa_{2}$ is the compressibility of the polymer solute, $\kappa_{\mathrm{s}}$, that of the solvation region, $v_{1}$ and $v_{2}$ are the specific volumes of the solvent and the solute, respectively, and $n_{\mathrm{s}}$ is the amount of solvent (expressed by gram) in the solvation per unit weight (gram) of solute. The method for obtaining $\bar{\kappa}_{2}{ }^{0}$ from the compressibility and density data of a dilute solution and the derivation of eq 3 are described in detail in our previous paper. ${ }^{16}$
The relative partial specific compression of a solute is defined by,

$$
K_{x}=\left(\frac{d-x}{d_{1}}-\frac{\kappa}{\kappa_{1}}\right) \frac{1}{x}
$$

where $d$ and $\kappa$ are, respectively, the density and compressibility of the solution, $x$ is the concentration of solute in grams per cubic centimeter of solution, and the subscript, one, refers to solvent. The quantity $K_{x}$ is related to $K$ defined by Shiio $e t$ al. ${ }^{11}$ as follows;

$$
K_{x}=K \frac{\kappa_{\mathrm{w}}}{\kappa_{1}}
$$

where $\kappa_{\mathrm{w}}$ refers to the compressibility of the water.

As was clearly shown in our previous works, $\bar{\kappa}_{2}{ }^{0}$ is a usefull parameter for describing the properties of solutions, especially interactions between solute and solvent. ${ }^{16}$ Moreover, in liquid-liquid mixtures where solute molecules do not interact with surrounding solvent, $\bar{\kappa}_{2}{ }^{0}$ agrees with the compressibility of the solute itself $\left(\kappa_{2}{ }^{0}=\kappa_{2}\right) \cdot{ }^{17}$

\section{EXPERIMENTAL}

\section{Samples}

The dextran samples used were supplied by Pharmacia Fine Chemical (Sweden) and are characterized in detail in our previous paper. ${ }^{15}$ The weight-average molecular weights were $1.0 \times 10^{4}$, $3.30 \times 10^{4}, 4.61 \times 10^{5}, 7.58 \times 10^{5}$, and $2.0 \times 10^{6}$. All saccharides used, i.e., glucose, maltose, and raffinose, were extra-pure reagents supplied from Nakarai Chemical Co., Ltd. They were dehydrated by procedures described previously. ${ }^{18}$ Dextran is hygroscopic so that before use, the samples were dehydrated in a vacuum electric oven at $90-95^{\circ} \mathrm{C}$ for several days.

The ethanol used was extra-pure grade reagent supplied from Hayashi Pure Chemical Industries, Ltd., and its water content was below $0.4 \%$. No further purification was carried out.

\section{Measurement}

The adiabatic compressibility of dextran solutions was determined by measuring the ultrasonic velocity and density. An improved interferometer working at $5 \mathrm{MHz}$ was used. The density was determined with an Ostwald type pycnometer of $20 \mathrm{~cm}^{3}$ capacity. 
Viscosity was measured with a Ubbelohde-type viscometer and the intrinsic viscosity $[\eta]$ was determined by the usual method.

All measurements were carried out at $25.00 \pm 0.03^{\circ} \mathrm{C}$.

\section{RESULTS AND DISCUSSION}

In a suspension system, the limiting partial specific compressibility, $\bar{\kappa}_{2}{ }^{0}$, should coincide with the compressibility of the suspensoid itself, $\kappa_{2}$.

To confirm this, we measured the ultrasonic velocities and densities of suspensions of monodisperse polystyrene latex (its diamerer, determined by dynamic light scattering, was about $4000 \AA$ ) as a function of the concentration of latex. The values of $\bar{\kappa}_{2}{ }^{0}$ and $\bar{v}_{2}{ }^{0}$ obtained were $26.8 \times 10^{-11} \mathrm{~Pa}^{-1}$ and $0.691 \mathrm{~cm}^{3} \mathrm{~g}^{-1}$, respectively. These values are in good agreement with the compressibility and reciprocal density $\left(v_{2}=1 / \rho\right)$ of polystyrene obtained by Maeda ${ }^{19}$ and Wada $^{20}$ for this polymer in the solid state.

Figure 1 shows plots of $\bar{\kappa}_{2}{ }^{0}$ against the ethanol concentration for a series of dextran samples. Similar curves were obtained for other samples investigated. The results for glucose are in good agreement with the literature data. ${ }^{12}$ With an in-

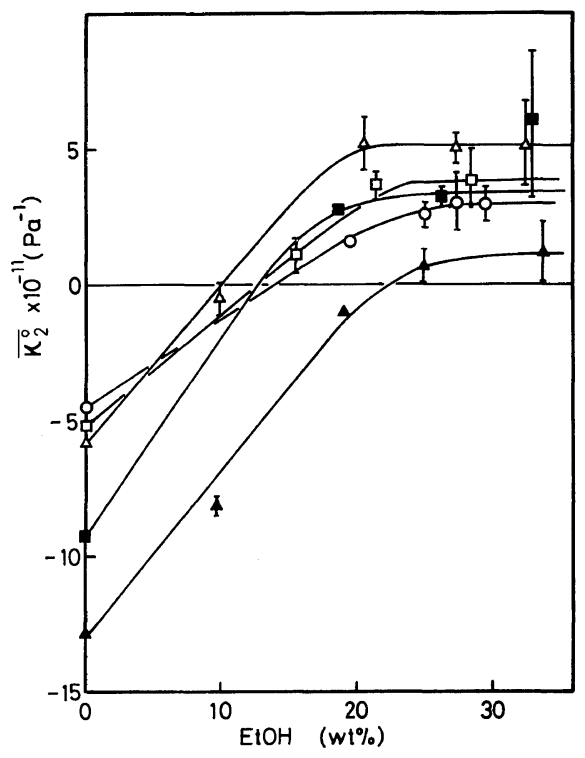

Figure 1. Effect of ethanol concentration on $\vec{\kappa}_{2}^{0}$ of dextran: $\boldsymbol{\Delta}$, glucose; $\square, \mathrm{T}-10 ; \triangle, \mathrm{T}-40 ; \square, \mathrm{T}-500 ; \mathrm{O}, \mathrm{T}-$ 2000 . crease in the ethanol concentration, the value of $\bar{\kappa}_{2}{ }^{0}$ increases and becomes constant at an ethanol concentration above $20 \mathrm{wt} \%$. This may be attributed to the partial transfer of bound water from solute molecules to solvent by ethanol. At ethanol concentration above $20 \mathrm{wt} \%$, the bound water of dextran was almost completely removed by ethanol. The value of $\bar{\kappa}_{2}{ }^{0}$ in this region may be represented only by the first term of eq 2 , that is, the compressibility of solute molecules, $\kappa_{2}$. The results obtained here indicate that the preferential solvation of water occurs for dextran as well as for glucose, maltose, and raffinose in water-ethanol mixtures.

The intrinsic viscosities of dextran decrease almost linearly with the ethanol concentration of a solvent, as shown in Figure 2. For higher molecular weight samples, the decrease in intrinsic viscosity is larger. This fact may also be due to the decrease in the bound water of dextran; as the ethanol concentration in solvent increases, the bound water is removed from the dextran chains. Therefore, the solvent becomes poorer for dextran as the ethanol content gets higher.

Although a breaking point was observed in the plots of $\bar{\kappa}_{2}{ }^{0}$ against the ethanol content, we found no clear breaking point in the relation between $[\eta]$ and the ethanol concentration (Figure 2). The decrease in $[\eta]$ with increasing ethanol content arises from the combined effects of dehydration and contraction of the polymer chain. The difference in the variation of $\bar{\kappa}_{2}{ }^{0}$ and $[\eta]$ with ethanol content shows that $[\eta]$ is mainly controlled by the expansion of the

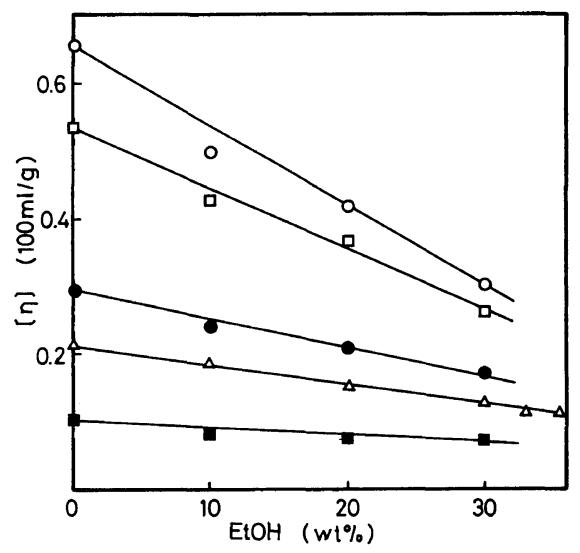

Figure 2. Effect of ethanol concentration on $[\eta]$ of dextran: $\square, \mathrm{T}-10 ; \triangle$, T-40; $\bigcirc$ T-70; $\square, \mathrm{T}-500 ; \bigcirc, \mathrm{T}-$ 2000 . 
polymer chain, while $\bar{\kappa}_{2}{ }^{0}$ is related to the local hydration or dehydration process.

Double-logarithmic plots of intrinsic viscosity against $M_{w}$ for dextran at various ethanol concentrations are shown in Figure 3. As shown in our previous paper, ${ }^{15}$ our results with aqueous solutions of dextran agree satisfactorily with those of Senti $e t$ $a l .{ }^{21}$ A downward deviation from the linear relation becomes larger with increasing ethanol concentration in the solvent. This behavior is similar to that in $40.5 \%$ aqueous methanol solution obtained by Wales et al. (the broken line in Figure $3),{ }^{22}$ and indicates that the dextran chains in solution become more closely packed with increasing molecular weight and decreasing solvent power.

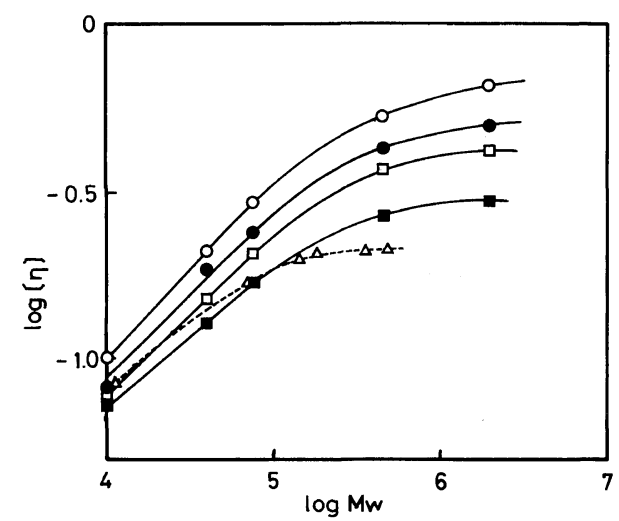

Figure 3. Double logarithmic plots of intrinsic viscosity, $[\eta]$, against $M_{w}$ for dextran at different ethanol concentrations: $O$, in aqueous solution; $O, 10 \%$ ethanol; $\square, 20 \%$ ethanol; $\square, 30 \%$ ethanol; $\triangle$, Wales et al. $^{22}$
The values of $\bar{\kappa}_{2}{ }^{0}$ and the partial specific volume, $\bar{v}_{2}{ }^{0}$, in an aqueous solution, the compressibility of solute molecule, $\kappa_{2}$, and the amount of bound water, $\omega\left(=n_{\mathrm{s}} v_{1}\right)$ estimated from eq 2 are tabulated in Table I. The value of $\kappa_{2}$ can be set equal to the value of $\bar{\kappa}_{2}{ }^{0}$ at the precipitation point.

As shown in Table $\mathrm{I}$, there is a reasonable decrease in the amount of bound water with molecular weight. The quantity $\kappa_{2}$ appears to decrease with increasing molecular weight, but not much significance can be placed on this behavior if we take experimental errors into account. At present, it seems appropriate to consider that the compressibility of dextran in solution is independent of the molecular weight. About $4.0 \times 10^{-11} \mathrm{~Pa}^{-1}$ is taken as the average compressibility of dextran.

The compressibility of dextran in an aqueous solution reported by Shiio et al. ${ }^{12}$ is $9.2 \times 10^{-11}$ $\mathrm{Pa}^{-1}$, which is twice as larger as that obtained here. This difference may be due to the different definitions of $K$ and the corresponding rough measurements for the concentration dependence of $K$. The compressibilities of maltose and raffinose in aqueous solutions are larger than that of glucose, but nearly equal to that of dextran. However, this does not seem reasonable.

Roughly, compressibility is equal to the reciprocal of elastic modulus. The method for calculating the elastic modulus of a polymer chain from its bond-stretching and -bending force constants was early developed and applied to cellulose by Meyer and Lotmar. ${ }^{23}$ Asahina et al. ${ }^{24,25,26}$ have presented an improved method for calculating elastic moduli of simple helical polymers on the basis of the Urey-

Table I. Partial specific compressibility, partial specific volume, compressibility of solute in solution, amount of bound water of glucose, maltose, raffinose, and dextrans

\begin{tabular}{|c|c|c|c|c|c|}
\hline \multirow{2}{*}{ Sample } & \multirow{2}{*}{$\begin{array}{l}\text { Molecular } \\
\text { weight }\end{array}$} & $\bar{\kappa}_{2}{ }^{0}$ & \multirow{2}{*}{$\frac{\bar{v}_{2}^{0}}{\mathrm{~cm}^{3} \mathrm{~g}^{-1}}$} & \multirow{2}{*}{$\frac{\kappa_{2}}{\times 10^{11} \mathrm{~Pa}^{-1}}$} & \multirow{2}{*}{$\frac{\omega}{\mathrm{cm}^{3} \mathrm{~g}^{-1}}$} \\
\hline & & $\times 10^{11} \mathrm{~Pa}^{-1}$ & & & \\
\hline Glucose & 180.16 & $-12.9 \pm 1.3$ & $0.623 \pm 0.002$ & 1.22 & 0.33 \\
\hline Maltose & 342.30 & $-8.0 \pm 0.7$ & $0.617 \pm 0.003$ & $5.2_{1}$ & 0.31 \\
\hline Raffinose & 594.50 & $-8.7 \pm 0.7$ & $0.612 \pm 0.002$ & 3.6 & 0.28 \\
\hline Dextran T-10 & $1.05 \times 10^{4 \mathrm{a}}$ & $-9.2 \pm 1.3$ & $0.604 \pm 0.000_{5}$ & 3.7 & 0.29 \\
\hline $\mathrm{T}-40$ & $3.30 \times 10^{4 \mathrm{a}}$ & $-5.8 \pm 0.4$ & $0.611 \pm 0.001$ & 5.1 & 0.25 \\
\hline $\mathrm{T}-500$ & $4.61 \times 10^{5 \mathrm{a}}$ & $-5.2 \times 0.4$ & $0.620 \pm 0.002$ & 3.7 & 0.21 \\
\hline T-2000 & $2.0 \times 10^{6 \mathrm{a}}$ & $-4.5 \pm 0.4$ & $0.614 \pm 0.002$ & $3.0_{0}$ & $0.17_{5}$ \\
\hline
\end{tabular}

\footnotetext{
a Molecular weight of dextran used is given in the data sheet.
} 
Bradley force field. We applied their improved method in estimating the elastic moduli of glucose, maltose, and dextran.

In order to calculate the elastic modulus, the structures and molecular dimensions of these molecules must be known. For glucose, the structure of $\alpha$-D-glucose in the cystalline state ${ }^{27}$ was used, and for maltose it was assumed that the $\mathrm{CH}_{2} \mathrm{OH}$ groups in glucose units take on gauche-trans conformation with each other. ${ }^{28}$ The higher hydrated structure of amylose in the crystalline state, that is, $V_{\mathrm{h}}$-form, has been shown by $\mathrm{X}$-ray diffraction to consist of helices having six glucose residues per turn, repeating at about $13.0-13.7 \AA$; this value is larger than $7.9 \AA$ in $V_{\mathrm{a}}$-form amylose, because of the inclusion of water molecules in the chain. ${ }^{29} \mathrm{We}$ assume that the local structure of dextran in solution has the same conformation as the $V_{\mathrm{h}}$-form of amylose.

Using the stretching and bending force constants of glucose, ${ }^{30}$ we obtained the elastic moduli of glucose, maltose, and dextran as $0.27_{4}, 0.74_{0}$, and $0.71_{0} \times 10^{-11} \mathrm{~Pa}^{-1}$, respectively. Even on taking into account of the rough assumption used, these values were found to be far from agreement with experimental ones. However, the order of their magnitude was consistent with that obtained in this work. Our results demonstrate the difficulty in calculating the compressibility of a polymer chain, this being a macroscopic quantity, from data on a microscopic level.

Acknowledgements. The authors should like to express their appreciation to Dr. S. Sasaki for supplying the mono-dispersed polystyrene latex samples. We are also thankful to Assistant Professor F. Kawaizumi of our laboratory for making measurements of the ultrasonic velocity and density of suspensions of polystyrene latex.

This work was supported in part by a grant from the Takeda Science Foundation.

\section{REFERENCES}

1. Y. Miyahara and H. Shiio, Nippon Kagaku Zasshi, 72, 876 (1951).

2. Y. Miyahara and H. Shiio, Nippon Kagaku Zasshi, 73, 1 (1952).
3. H. Shiio, J. Am. Chem. Soc., 80, 70 (1958).

4. K. Gekko and H. Noguchi, Macromolecules, 7, 224 (1974).

5. Y. Suzuki and H. Uedaira, Bull. Chem. Soc. Jpn., 43, 1892 (1970).

6. H. Noguchi, Adv. Polym. Sci. Jpn., 8, 191 (1975).

7. Y. Miyahara, Bull. Chem. Soc. Jpn., 29, 742 (1956).

8. Issak E. Élpiuer, "Ultrasound, physical, chemical and biological effect," Consultants Bureau, New York, 1964, Chapter 7.

9. R. J. Urick, J. Appl. Phys., 18, 983 (1947).

10. K. Fukutomi and Y. Wada, J. Acous. Soc. Jpn. (in Japanese), 15, 22 (1959).

11. H. Shiio, Nippon Kagaku Zasshi, 74, 203 (1953).

12. H. Shiio, T. Ogawa, and H. Yoshihashi, J. Am. Chem. Soc., 77, 4980 (1956).

13. K. Itoh, Nippon Kagaku Zasshi, 77, 1594 (1956).

14. H. Nomura, S. Yamaguchi, and Y. Miyahara, $J$. Appl. Polym. Sci., 8, 2731 (1964).

15. F. Kawaizumi, N. Nishio, H. Nomura, and $Y$. Miyahara, Polym. J., 13, 209 (1981).

16. H. Nomura and Y. Miyahara, J. Appl. Polym. Sci., 8, 1643 (1964).

17. H. Nomura and Y. Miyahara, Nippon Kagaku Zasshi, 89, 145 (1969).

18. F. Kawaizumi, N. Nishio, H. Nomura, and Y. Miyahara, J. Chem. Thermodynamics, 13, 89 (1981).

19. Y. Maeda, J. Appl. Phys. Jpn. (in Japanese), 26, 22 (1957).

20. Y. Wada, J. Appl. Phys. Jpn. (in Japanese), 24, 159, 287 (1955).

21. F. R. Senti, N. N. Hellman, N. H. Ludwig, G. E. Babcock, R. Tobin, C. A. Glass, and B. L. Lamberts, J. Polym. Sci., 17, 527 (1955).

22. M. Wales, P. A. Marshall, and S. G. Weissberg, $J$. Polym. Sci., 10, 229 (1953).

23. K. H. Meyer and W. Lotmar, Helv. Chim. Acta, 19, 68 (1936).

24. T. Shimanouchi, M. Asahina and S. Enomoto, $J$. Polym. Sci., 59, 93 (1962).

25. M. Asahina and S. Enomoto, J. Polym. Sci., 59, 101 (1962).

26. S. Enomoto and H. Asahina, J. Polym. Sci., 59, 111 (1962).

27. G. M. Brown and H. A. Levy, Science, 147, 1038 (1965).

28. C. V. Goebel, W. L. Dimpfl, and D. A. Brant, Macromolecules, 3, 644 (1970).

29. J. J. Cael, J. L. Koenig, and J. Blackwell, Carbohyd. Res., 29, 123 (1973).

30. P. D. Vasko, J. Blackwell, and J. L. Koenig, Carbohyd. Res., 23, 407 (1972). 